\title{
Embedding Thinned Chips in Flexible PCBs
}

T. Sterken ${ }^{1}$, F. Vermeiren ${ }^{1}$, P. Tremlett ${ }^{2}$, W. Christiaens ${ }^{3}$, J. Vanfleteren $^{1}$

${ }^{1}$ CMST (Affiliated with IMEC and Universiteit Gent), Technologiepark 914a, B-9052 Gent, Belgium

${ }^{2}$ Microsemi, Caldicot, Monmouthshire, NP26 5YW, UK

${ }^{3}$ ACB nv, Vosmeer 3, B-9200 Belgium

Tel: 0032- 92645514, Fax: 0032- 92645374, Email: Tom.Sterken@imec.be

\begin{abstract}
Autonomous systems for healthcare and sports applications benefit largely from a small form factor, as minute dimensions result in maximal mobility and comfort. By embedding a commercially available IC in a flexible circuit board (FCB), the overall size of a system can be reduced. This work presents the process of thinning and packaging an IC in a flexible interposer, and the embedding thereof in a FCB. To illustrate the potential of this technology towards miniaturization, a commercially available RF transceiver ZL70102 was packaged and embedded, thus realizing a volume reduction of $60 \%$.
\end{abstract}

\section{Introduction}

Research in the field of healthcare and sport monitoring tends to focus on the development of smart devices, where the output of multiple sensors is combined with the functionality of a microcontroller, memory and an RF transceiver for wireless communication to a base station or other nodes in the Body Area Network (BAN). The picture is often completed with a battery or energy harvester, thus providing an autonomous solution which enables the user or patient to monitor his/her health with a higher effectiveness and precision. Conventional technology often results in bulky solutions, and miniaturization of these systems only seems to be possible at the expense of functionality, autonomy and comfort.

This paper reports on the development of a versatile packaging technology which allows integrating commercially available ICs into a miniature package by embedding the chip into a flexible circuit board (FCB). This action then allows rerouting the passives on top of the IC, which results in a system with a smaller form factor, breaking the boundaries imposed by SMD technology and fine pitch packages.
This work presents the basic elements of integrated thinned dies into a FCB, by first embedding the thinned die into an ultra-thin chip interposer package(UTCP). Next, one or multiple UTCPs are embedded into the FCB using a stateof-the-art technology which is available in commercial PCB foundries. This two-step approach offers the possibility of checking the yield of the ultra-thin chip packages before embedding in order to eliminate the failures and reduce the costs. As a demonstrator a medical RF transceiver is thinned and embedded into a 4-layer FCB, thus realizing a volume reduction of $60 \%$.

\section{UTCP interposer}

Our research activities on embedding thinned dies into a polyimide package were first presented in 2006 [1], when a microcontroller was embedded between two layer of nonphotopatternable spin-on polyimide PI-2611 of HD Microsystems 22. Later on this package was used for embedding in a flexible circuit board 3. Since, our research on both packaging thinned dies in flexible interposers and the embedding thereof in flexible circuit boards, has evolved towards a higher compatibility of the process to available industrial standards, by increasing the yield and production capacity of the process while trying to reduce the processing time and costs. A summary of the process flow used in this work is given in figure 1 . As the process flow has been described in detail in the following publications 4, 5,6, we will however restrict ourselves to highlighting the most significant changes since 2006 :

\section{> Photopatternable polyimide}

The polyimide PI-2611 is a non-photopatternable polyimide. The vias in the top layer are therefore created either using laser ablation [1], which is a serial process, either using RIE etching [6], which has a limited etch rate of $200 \mathrm{~nm} / \mathrm{min}$.
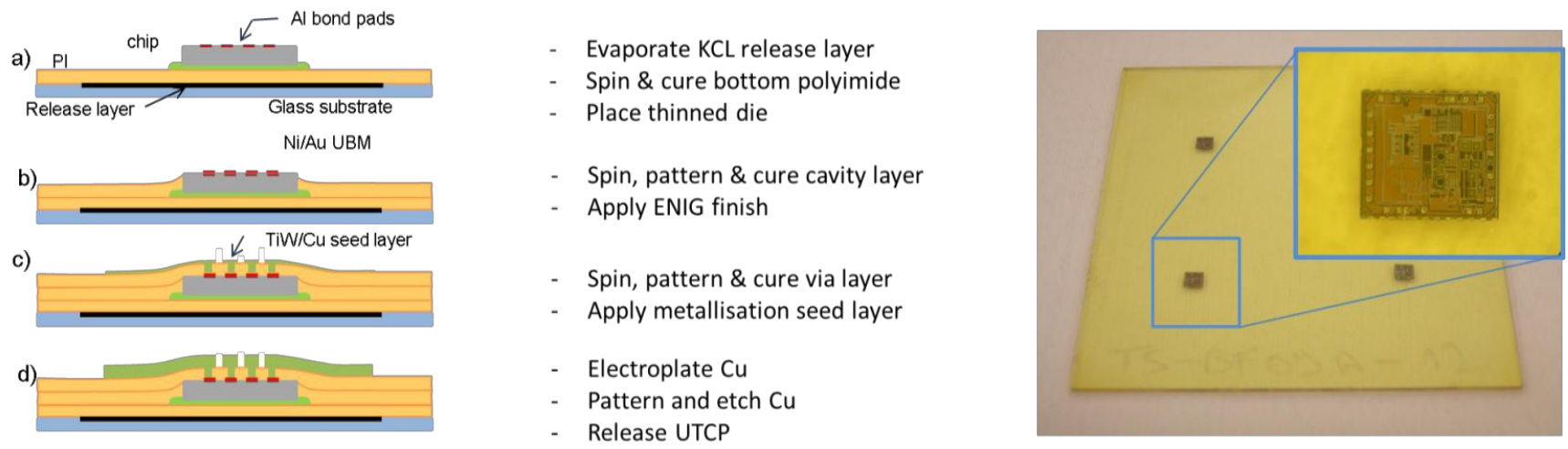

Figure 1 : Schematic overview of the process flow (Left) and a photograph of a substrate with 4 thinned ZL70102 ICs placed on the bottom polyimide (Right). 
Furthermore, the thickness of a single layer of PI2611 is limited to about $5 \mu \mathrm{m}$ due to its low viscosity, which results in 4 spin sessions and 2 curing cycles in order to obtain a layer with a thickness of $20 \mu \mathrm{m}$.

By using photosensitive polyimide HD-4110 8, only one spinning and curing cycle is needed to achieve the desired thickness, while the layer can be structured using a mask aligner. When multiple ICs are packaged on the same processing substrate, a stepper mask approach is used.

\section{$>$ Releaselayer}

The self-priming nature of HD-4110 requires the use of an additional release layer. In this work, we use a thermally evaporated layer of $400 \mathrm{~nm}$ of $\mathrm{KCl}$ salt, which can be dissolved in water during the final release step. The salt can withstand the high curing temperatures of the polyimide.

By patterning the salt with a shadow mask during evaporation, multiple ICs are packaged simultaneously on the same substrate: the mechanical stability of each polyimide membrane during processing is ensured.

\section{$>$ Cavity layer}

A third layer of polyimide is introduced in between the top and bottom layer. This layer is patterned using backside illumination, thus embedding the thinned die in a self-aligned cavity. The advantage of this cavity layer is three-fold:

1. The step coverage of the top polyimide at the edge of the chip is ensured, thus preventing short-circuits by isolating the metal fanout from the edge of chip

2. The topography of the finished UTCP is reduced, a so-called "flat UTCP" is created. Not only does this result in a better lithography in the subsequent processing steps: flat UTCPs are necessary to improve the yield when the packages are used for 3D stacking [6].

3. Side-wall isolation is created. This allows applying an electroless nickel-gold finish (ENIG) on the contact pads of the IC with a limited amount of skipping pads, even during the process of UTCP packaging.

The result of the process given in Figure 1 is a flexible interposer with a thickness of about $70 \mu \mathrm{m}$, mounted on a glass carrier. The design of the metal fanout allows converting the fine pitch (e.g. $60 \mu \mathrm{m}-100 \mu \mathrm{m}$ ) of the IC to a larger pitch compatible with the intended application, such as embedding or stacking. Furthermore, it is possible to create interconnections between several contact pads of the IC.

Before the UTCP is released from its processing carrier, a basic functionality test is performed: at first the quality of the interconnection to the chip is verified by measuring the ESD diode threshold voltage. In some cases a full functionality test is possible using a probe card. In [4], the yield of packaging microcontrollers was measured to be $87 \%$, while a DSP processor was packaged with a yield of $62 \%$.

Based on these tests one can assume having Know-GoodPackages.

\section{Prototypes}

In this work we present the packaging and embedding of a ZL70102 RF transceiver for medical implantable applications, provided by Microsemi [9]. The bare die has a dimension of $4275 \times 3145 \mu \mathrm{m}^{2}$, on which 70 bondpads of $80 \times 80 \mu \mathrm{m}^{2}$ are placed. This dies is embedded in an FCB with the aim of minimizing the overall volume of the radio module, including the passives on the FCB.

A second prototype consists of a MSP430F1611 microcontroller with an area of $4630 \times 4450 \mu \mathrm{m}^{2}$, with 64 bondpads of $75 \times 75 \mu^{2}$. As these ICs can be used for a variety of applications, a general fan-out is used. (a)

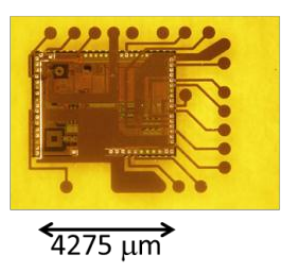

(b)

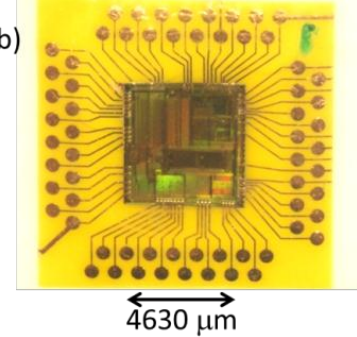

Figure 2 : Photograph of the released ultra-thin chip packaged Z170102 (a) and MSP430F1611(b)

\section{UTCP embedding}

The embedding of the UTCP into a flexible circuit board is based on a commercially available process, and performed by ACB nv [10]. In this process multiple copper cladded sheets (Pyralux series by Dupont) are laminated using the LF0100 sheet adhesive by the same provider. Prior to lamination, the UTCP package is aligned to a patterned inner layer (figure 3, (a)), and fixed to this position by locally heating the adhesive (e.g. using a soldering iron). A second adhesive sheet is inserted on top of the UTCP. The stack is next laminated (fig. 3b) and the FCB is finished by drilling through-holes, patterning of top and bottom metal layers and electroplating the vias.

(a)

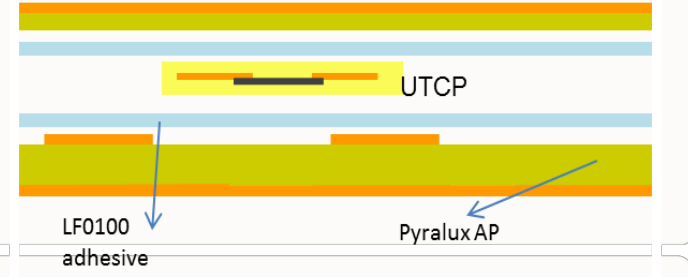

(b)

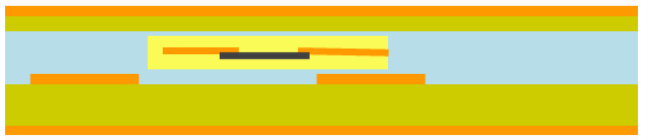

(c)

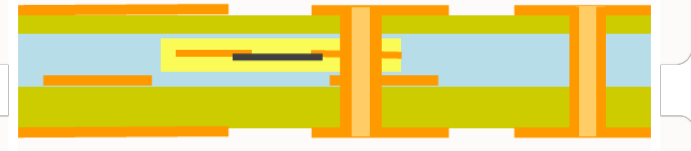

Figure 3 : Overview of the process flow for embedding a UTCP in an FCB during lamination. 


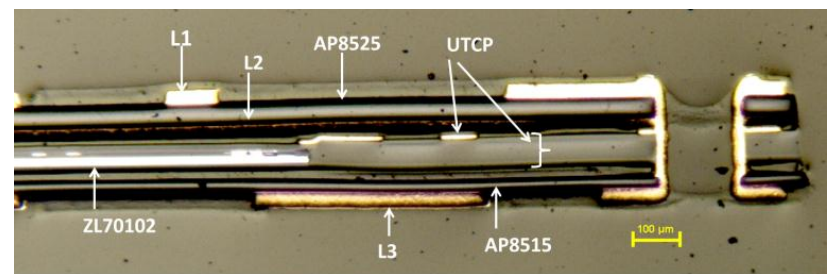

Figure 4 : Cross-section a UTCP embedded in a three-layered FCB. On the right side a through-hole via connects all 4 metal layers.

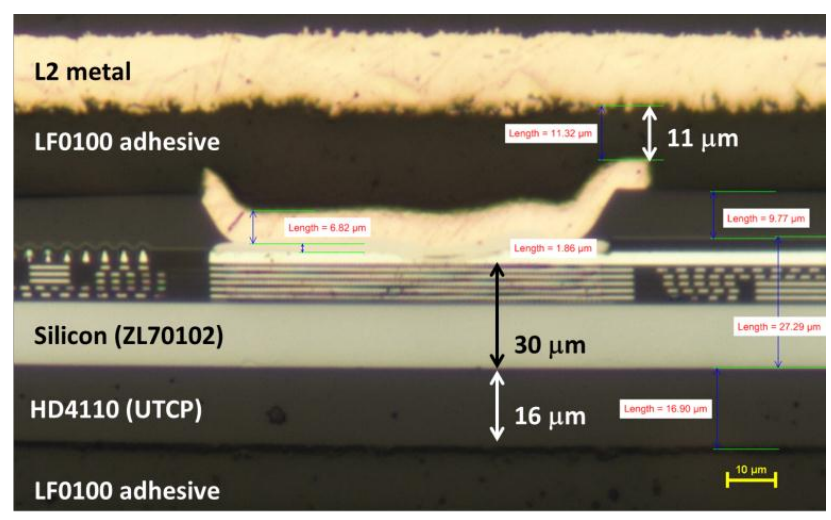

Figure 5 : A closer view of the UTCP illustrates that the chips has been thinned up to $30 \mu \mathrm{m}$, while the UTCP is isolated from the inner metal layer L2 by $11 \mu \mathrm{m}$ of LF0100 adhesive.

The overview of the process flow in figure 3 refers to a 3 layer FCB, composed by laminating a double-sided polyimide substrate AP8525 to a single-sided substrate AP8515. This approach was used for packaging the ZL70102, while the microcontroller was embedded in a 4-layered flex. The latter is composed of one sheet of AP8525, sandwiched in between two layers of AP8515. After embedding the KGD microcontrollers, $95 \%$ of the ICs were still fully operational. The failure modes that occurred were traced back to misalignments of the UTCP package to the inner layers of the FCB.

\section{Cross-sections}

Figure 4 illustrates a cross-section of the radio transceiver embedded in the 3-layer FCB. The total thickness of the flex averages around $250 \mu \mathrm{m}$. The image also illustrates that a through-hole via connects all 3 copper layers as well as the UTCP metal.

In figure 5 the double-sided AP8525 flex is located on top of the UTCP. As a result, the inner copper layer L2 is facing the copper of the UTCP. Both layers are only isolated from each other by the LF0100 adhesive. Nevertheless, both layers are still separated by about $11 \mu \mathrm{m}$. Although this issue didn't result in failure, it is a good design rule to avoid facing copper planes between the UTCP and FCB inner layers.

\section{Yield}

In this work 32 microcontrollers were packaged in a UTCP package, of which $87.5 \%$ were proven to be functional. The functionality was derived based on the possibility of programming the MSP430. The embedding of these UTCP packages proved to be possible at a yield of $95 \%$, based on the same full functionality tests.

For the UTCP-packaged Radio ICs, a yield of $85 \%$ is measured based on the measurement of the ESD-diodes of the contact pads. A full functionality test was however not possible at this stage. After embedding the dies in the FCB however, none of the 22 dies seems to qualify. Based on the high yield experienced in embedding the microcontrollers on an FCB, it can be assumed that the ESD-diode test did not provide the correct information for solving the KGD-problem. At the moment of writing, the research of these failure modes is still ongoing.

\section{Size reduction and applications}

Figure 6 illustrates the volume reducing power of embedding ICs in the circuit board. In figure $6 \mathrm{a}$ the microcontroller is packaged in a conventional plastic quad flatpack (QFP), and placed on a reference FCB. The total volume occupied by the package, is about $240 \mathrm{~mm}^{3}$, while it occupies about $150 \mathrm{~mm}^{2}$ of the FCB. The thickness of the FCB is $300 \mu \mathrm{m}$ (4-layered FCB). The UTCP-packaged microcontroller occupies an area of $1.1025 \mathrm{~mm}^{2}$ while the FCB thickness increased to $350 \mu \mathrm{m}$. The use of embedded UTCP packages allows recycling about $50 \%$ of the occupied area for placing passives and routing.

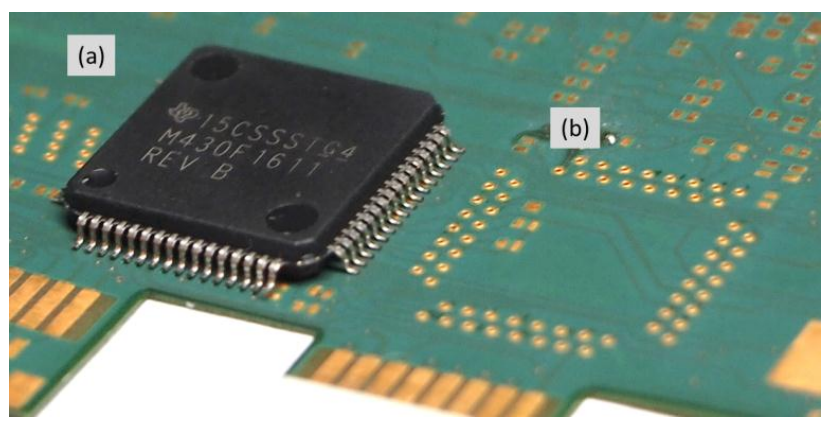

Figure 6 : A conventionally QFP-packaged MSP430F1611 (a) is compared to an embedded UTCP-packaged version (b).
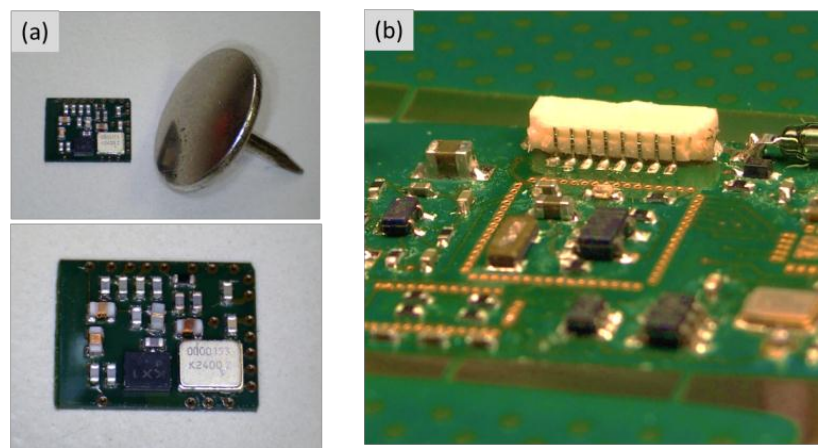

Figure 7 : The embedding of the die in the FCB not only allows reducing the thickness of the component, the area of the die can be reused for placing passives. On the left side the ZL70102 is embedded, while the image on the right side illustrates the routing of components on a MSP430F149, embedded in a FCB. 


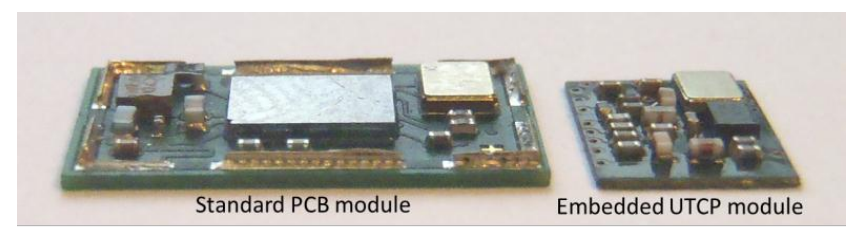

Figure $8:$ On the left side a system based on a CSP packaged ZL70102 is compared to its replica based on an embedded UTCP.

Note that the vias to the UTCP also occupy a considerable amount of area. Limiting the amount of $\mathrm{I} / \mathrm{O}$ to the bare necessity will further enhance the miniaturizing effect of embedded ultra-thin chip packages. Figure 7 illustrates how many components can be mounted on top of embedded UTCPs using standard reflow soldering techniques. Figure 8 illustrates in one image the power of UTCP for minimizing small electronic systems. The total volume of the UTCPbased module is $<60 \%$ of the original module.

\section{Conclusion}

Embedding dies in a circuit board is a promising way of miniaturizing electronic systems for healthcare and sports applications. Both a MSP430F1611 microcontroller and a ZL70102 RF transceiver are packaged in an Ultra-Thin Chip Package, which are subsequently embedded in a flexible circuit board. The yield of embedding an IC in an ultra-thin chip package reaches up to $85 \%$, while the embedding of the UTCP into an FCB was successfully performed for the MSP430F1611. The use of embedded UTCPs allows in this way to use about $50 \%$ of the footprint area of a conventional package for placing passives and for routing.

\section{References:}

1. W. Christiaens, E. Bosman and J. Vanfleteren, "A Novel Polyimide-Based Ultra-Thin Chip Packaging Technology", IEEE transactions on components and packaging technologies, vol. 33, no. 4, 2011, pp. 754-760

2. PI-2611, datasheet provided by HD microsystems, curently available at :

http://hdmicrosystems.com/HDMicroSystems/en_US/pdf/ PI-2600_ProcessGuide.pdf

3. W. Christiaens, T. Torfs, W. Huwel, C. Vanhoof, J. Vanfleteren, "3D integration of ultra-thin functional devices inside multilayer standard flex laminates", European Microelectronics Packaging Conference (EMPC), 2009

4. T. Sterken, M. Op de Beeck, F. Vermeiren, T. Torfs, L. Wang, S. Priyabadini, K. Dhaenens, D. Cuypers and J. Vanfleteren "High Yield Embedding of $30 \mu \mathrm{m}$ Thin Chips in a Flexible PCB using a Photopatternable Polyimide based Ultra-Thin Chip Package (UTCP)", Proc. 45th International Symposium on Microelectronics (IMAPS 2012), San Diego, CA

5. L. Wang, T. Sterken, M. Cauwe, D. Cuypers and J. Vanfleteren, "Fabrication and Characterization of Flexible Ultrathin Chip Package Using Photosensitive
Polyimide", IEEE Transactions on Components, Packaging and Manufacturing technology, Vol.2, no. 7, 2012, pp. 1099-1106

6. S. Priyabadini, T. Sterken, L. Wang, K. Dhaenens, B. Vandecasteele, S. Van Put, A.E. Petersen and J. Vanfleteren, "An approach to produce a stack of photo definable polyimide based flat UTCPs", Proc. $4^{\text {th }}$ Electronic Systems Integration Technologies Conference (ESTC), 2012

7. R. Verplancke, T. Sterken, D. Cuypers and J. Vanfleteren, "Thinned Dies in a Stretchable Package", Proc. $4^{\text {th }}$ Electronic Systems Integration Technologies Conference (ESTC), 2012

8. HD-4110, datasheet provided by HD microsystems, curently available at :

http://hdmicrosystems.com/HDMicroSystems/en US/pdf/ HD-4100 ProcessGuide.pdf

9. Product information is currently available at : http://www.zarlink.com/zarlink/hs/82_ZL70102.htm

10. Company website :http://www.acb.be/ 